\title{
Evaluation of distributed DNA representations on the classification of conserved non-coding elements
}

\author{
Nikolaos Gialitsis \\ nikolasyal@di.uoa.gr \\ NKUA
}

\author{
George Giannakopoulos \\ ggianna@iit.demokritos.gr \\ IIT, NCSR-D
}

\author{
Marina Athanasouli \\ marina.athanasouli@tuebingen.mpg.de \\ MPI-EB
}

\begin{abstract}
The representation of DNA sequences has been an interesting topic of discussion for many years. Presently, given the usefulness of representations built upon embeddings for Natural Language Processing (NLP), there have been efforts to transfer such paradigms to the DNA world and related problems. In this paper, we study different DNA representations on the well-studied problem of Conserved Non-coding Elements (CNEs), trying to understand how well existing representations utilize the value of context, both in terms of local, near context, but also of long-distance interactions in genomic sequences. To this end, we apply a number of methods, including probabilistic models (LDA) and hybrid probabilistic-neural models (lda2vec) on appropriate datasets, compare the results to pre-existing methods and discuss the findings to better understand the value and challenges of different representations in the given domain.
\end{abstract}

\section{ACM Reference Format:}

Nikolaos Gialitsis, George Giannakopoulos, and Marina Athanasouli. 2020. Evaluation of distributed DNA representations on the classification of conserved non-coding elements. In 11th Hellenic Conference on Artificial Intelligence (SETN 2020), September 2-4, 2020, Athens, Greece. ACM, New York, NY, USA, 7 pages. https://oi.org/10.1145/3411408.3411463

\section{INTRODUCTION}

With the rise of Biomedical Natural Language Processing (BioNLP) many problems in the biomedical world have been transformed into NLP problems. Since DNA is read as a sequence of symbols (nucleotides), a genetic sequence can easily be considered equivalent to a natural language text, made up from the limited alphabet $\{A, T, C, G\}$. Similarly, the alphabet for protein sequences typically consists of 20 unique letters, one per amino acid (e.g "M" for "Methionine"). As follows, computational linguistics have been applied very successfully in the biomedical world for tasks aiming to support sequence matching [20], indexing [21], analysis of proteomic sequences [9] and coding and non-coding DNA sequences [25, 35], protein classification $[7,40]$, and phylogenetic tree reconstruction [37].

DNA words are typically produced by a sliding window of $k$ letters through the length of the whole sequence. In the literature, these words are often referred to as k-mers. In the following

\section{(c) (i)}

This work is licensed under a Creative Commons Attribution International 4.0 License.

SETN 2020, September 2-4, 2020, Athens, Greece

(C) 2020 Copyright held by the owner/author(s).

ACM ISBN 978-1-4503-8878-8/20/09 .. \$15.00

https://doi.org/10.1145/3411408.3411463 sections we will follow the convention of using the "k-mer" terminology when referring specifically to DNA sequences and the "word" terminology when referring to more general concepts.

The aim of this study is to summarize and extend the arsenal of DNA representation methods found in the literature, and evaluate the importance of local and global contextual information in DNA representations.

\section{RELATED WORK}

\subsection{Sparse representations}

Traditionally, k-mers are represented with an one-hot-encoding. The dimension of each vector is equal to $4^{k}$ which hinders model learning due to the curse of dimensionality [4]. Furthermore, the orthogonality of one-hot vectors does not reflect word-similarity. More memory-friendly representations one-hot-encode each nucleotide as a vector of size 4 such as the one proposed by Voss et.al [43], but that approach also considerably increases the input's dimensionality.

In contrast to recent embeddings which have roots in Natural Language Processing or Graph Theory [31, 35, 42], nucleotide embeddings based on biochemical aspects have also been proposed. These embeddings map each nucleotide to a vector of one or multiple dimensions where each dimension reflects a natural attribute. For instance, one-dimensional approaches include mapping nucleotides to their atomic numbers proposed by Holden et al.[16] or to their electron ion pseudo-potential (EIP), a metric related to the energy of electrons [29]. A similar approach called 'PairedNumeric', assigns $\{A=C=+1, T=G=-1\}$ in order to take advantage of the complementary nature of nucleotides [2] .

Therefore, a straightforward approach to construct the embedding for a genomic sequence is by concatenating all singular nucleotide vectors. Notably, this introduces additional computation and memory overhead, as it often produces sequence length-dependent representations with very high dimensionality. Nowadays, more advanced methods are preferred, but one has to keep in mind that these consisted the first step towards the numeric representation of biological entities. Another major disadvantage is that padding is needed in order to represent sequences of variant length which can negatively impact model learning.

\subsection{Distributed representations}

Recent approaches have adopted word2vec [27], a 2-layered neural network that strives to learn word-representations from their surrounding words. This reconstruction of words' context is loosely inspired by the linguistic concept of distributional hypothesis, which states that words appearing in the same context have similar meaning [15]. 
dna2vec. In dna2vec [31], non-overlapping k-mers are extracted from a long input sequence with a variable $k$, sampled with equal probability from a pre-defined interval. A word2vec model is trained on the k-mers producing 100-dimensional embeddings. The paper demonstrates that vector addition is analogous to nucleotide concatenation. Additionally, their results provide evidence that the cosine similarity of the resulting vectors is highly correlated with the Needleman-Wunsch similarity score for global sequence alignment (based on dynamic programming) [30]. These k-mer embeddings appear more sensible and robust than their one-hot-vector encoded counterparts but are still higher-dimensional than the input genomic string. Furthermore, word2vec outputs are extremely hard to interpret or to visualize as they tend to be very dense.

Nucl2vec. Another similar approach, Nucl2vec [10] trains word2vec on k-mers with the goal of aligning a small query sequence to a long reference genome. Due to the fact that the query might not match exactly with the reference due to natural divergence and sequencing errors, Ganesh et al. modify word2vec to accommodate for insertions and substitutions. For each k-mer in the query, they construct a new sentence by initially positioning the k-mer in the middle of the sentence. Next, they construct the k-mers' neighborhood by forming all possible strings with edit-distance one from it, simulating insertions and substitutions. For the alignment itself, they used the k-nearest neighbor algorithm which achieved accuracy comparable with state-of-art methods.

Nucl2vec examines 1-dimensional and 2-dimensional embeddings on the task, in contrast to 100-dimensional vectors produced in dna2vec. However, as reported in the same study, the euclidean distance between the projected k-mers is not always analogous to their edit distance. Thus, this approach reduces computational time at the cost of representation efficiency.

Autoencoders. In addition to word2vec-based approaches, Agarwal et al. [1] recently utilized Autoencoders to produce latent representations from DNA sequences. Autoencoders are artificial neural networks that learn representations in two successive phases: (1) they encode the input into fixed-length latent embeddings and (2) they decode the embeddings back to the input trying to minimize information loss. Agarwal et al. proposed an autoencoder consisting of a bi-directional LSTM as the encoding layer and an unidirectional LSTM as the decoding layer to learn efficient DNA representations [1]. Application of this methodology on the task of splice site prediction demonstrated that autoencoder representations, when used as priors for the initialization of neural network weights, enhance classification accuracy and convergence speed compared to a random initialization.

Although this is a promising approach, it still encapsulates the core issue of DNA representation. During training of the autoencoder, right before the encoding step, the DNA sequences are fed into an embedding layer which represents each nucleotide with a one-hot-encoding. This non-distributed intermediate representation can be both memory and time inefficient especially when dealing with long DNA sequences. Furthermore, we have already mentioned alternative methods for the numeric representation of nucleotides (2.1) such as Paired-Numeric or the Electron-Ion Potential representations [2,29] which are lower-dimensional and are based on biochemical properties.
2.2.1 Distributed representations from graphs. Graph embeddings have also been applied to represent genomic sequences. The sequence is initially transformed into a graph where each node corresponds to a k-mer and an edge connects two adjacent k-mers. Random walks can be used to generate contexts for each node, and then embed them into a real vector space with word2vec.

Multi-lateration. Tillquist and Lladser [42] took advantage of the notion of multi-lateration and the properties of Hamming graphs to produce low-dimensional k-mer embeddings. Multi-lateration is a method to represent a point in space by its distance to a set of reference points, called resolving set. Determining the minimal resolving set, which would result into the lowest-dimensional embedding of the sequences, is NP-complete for general graphs. By using Hamming graphs, this restriction is bypassed and low-dimensional graph embeddings can easily be derived. They tested their pipeline on a binary classification experiment involving genomic sequences (intronexon boundary prediction), and they produced 108-dimensional vectors to represent 20 -mers. This approach guarantees unique distinguishable embeddings which do not require rebuilding the graph from scratch when considering new k-mers. Furthermore, since rich literature exists in graph-theory methods with rigorous mathematical foundations, graph-embeddings appear promising for DNA-representation.

$N$-gram graphs. Another graph-based method that has been applied for DNA representation which does not utilize word2vec is the N-gram graph embedding [12]. N-gram graphs (NGGs) have attributes that allow the identification of both local and global characteristics of the analysed sequences. The main idea behind NGGs is that the statistics of neighbouring sub-sequences in a sequence contain a crucial part of the sequence information. Thus, a sequence is split in overlapping subsequences (n-grams), which are considered to co-occur when found within a maximum distance threshold of each other. These neighbours are represented as connected vertices in a graph. The edge connecting the neighbours can be weighted in several ways, the most common case being the number of cooccurrences within a given window size. For classification, a graph is constructed for each query sequence, as well as for each candidate class during training. The similarity vectors are calculated between the query-graph and the class-graphs, thus projecting the query sequence into a $C$-dimensional space of similarities where $C$ is the number of classes. NGGs have successfully been applied for the classification of conserved elements and nucleosome locations $[22,35]$.

\subsection{Genomic Signatures (GS)}

Genomic Signature representations are an established approach on representing genomic sequence data $[17,19,36]$. GS are based on the observation that the dinucleotide odds ratios (e.g 'AT' ratio) tend to be the same among organisms of the same species, and closely related organisms have substantial more similar dinucleotide ratios than those distantly related. Thus, these ratios can be thought to have a 1-to-1 relationship with species' genomes. Genomic Signatures are an effective method of discriminating between sequences from different organisms. 
Table 1: Summarizing information on DNA embeddings found in the literature

\begin{tabular}{|c|c|c|}
\hline Method & Tokenization & Feature Dimensionality \\
\hline multi-lateration[42] & 20-mers & 108 \\
\hline dna2vec[31] & $k$-mers, $k \in[3,8]$ & 100 \\
\hline Genomic Signatures [35] & 5-mers & 16 \\
\hline N-gram graph[22, 35] & 5-mers, 3-mers & 2,6 \\
\hline Nucl2vec[10] & 4-mers & 1 \\
\hline
\end{tabular}

With GS, DNA sequences can be represented as 16-dimensional embeddings $\left(4^{2}\right)$, where each dimension corresponds to the sequences' odds ratio of a specific dinucleotide. The GS method has been effectively applied by Polychronopoulos et al. [35] in order to produce DNA embeddings for the classification of diverse genomic sequences, including conserved non-coding elements.

\subsection{Topic Modeling}

2.4.1 Latent Dirichlet Allocation (LDA). Topic models can infer topics by observing the distribution of words across documents. This can be accomplished with Latent Dirichlet Allocation (LDA) $[5,41]$, a generative statistical model that makes the hypothesis that there exists an underlying distribution of words, topics and documents, which generated the input text collection. Using probabilistic topic model jargon, the words of a document are called "observed variables", whereas the variables of the topic structure are called "hidden variables". Using an iterative process, the model estimates the posterior distribution of the hidden variables given the observed variables. However, the vast amount of topic structures that can exist result in exponential complexities of computation. For this reason, sampling-based algorithms have been developed, such as Gibbs sampling.

2.4.2 Gibbs sampling. In Gibbs sampling [11, 41], a Markov chain (i.e., a sequence of random variables, each only dependent on the previous) is constructed, using samples from the distribution of hidden variables. The assignment of words to topics is sampled iteratively until the Markov chain converges to the target distribution. In the beginning of this procedure, each word is randomly assigned to a topic and in each subsequent iteration, the word-topic assignments are re-evaluated, which might result in words passing through multiple topics during the process.

\section{5 lda2vec}

lda2vec [28] aims to combine the flexibility of word2vec with the interpretability of LDA. Moreover, since word2vec is efficient at capturing local-relationships while LDA excels at identifying global document-level features, lda2vec is expected to provide richer and more context-aware representations than word2vec.

lda2vec takes advantage of the parallelization capabilities and speed of modern GPUs as well as of the computationally effective matrix-factorization formulation of word2vec proposed by Levy and Goldberg [24] to train word-embeddings as well as extract document-topic vectors.

The skip-gram model with negative sampling is employed by lda2vec in similar fashion to word2vec. However, when predicting a pivot word's context during model training, addition is performed between the pivot's word vector and the document vector. The intuition is that some words will be preferred more than others if we consider the topic-composition of a document. For example, in natural language, the context for the pivot word "English" without any topic-consideration might contain any of the following \{"language", "history", "lessons","tea"\}. On the other hand if we know the document is about the Olympics, then context-words like \{"team", "athlete" , "wins"\} might be more sensible choices.

lda2vec produces sparse and low-dimensional document-vectors which are more interpretable than the dense representations of word2vec. Sparsity is enforced by a loss function, which restraints the documents to express as few topics as possible. The document vectors are passed through a softmax function in order to correspond to probabilities. All the values of the output document vector always sum to one and belong in the $[0,1]$ interval. A disadvantage of lda2vec is that it takes considerably more time to execute than its respective counterparts, word2vec and LDA. [28]

\section{MOTIVATION}

Most approaches found in the literature for DNA representation, produce dense and high-dimensional vectors which focus on local relationships between k-mers. Thus, the importance of long-range interactions between k-mers is unaccounted for or under-utilized. In genomics especially, regulatory genes affect the transcription (i.e the construction of RNA from template DNA) of other genes that are several bases apart [32, 34, 38]. This shortcoming is alleviated by the application of the lda2vec model, which expands on word2vec by producing topic-aware word vectors from a combination of local and global information. In this paper, we explore whether high-level contextual information can be used to produce efficient distributed embeddings. To assess this, we perform multiple classification experiments on genomic sequences that exhibit a very high degree of similarity. To our knowledge, it is the first time that LDA and lda2vec have been applied to the classification of conserved non-coding elements.

\section{PROPOSED METHOD}

\subsection{Classification of CNEs}

4.1.1 Theoretical Background. Comparative genomics led to the identification of highly conserved non coding elements (CNEs) between distantly related vertebrates that appear to be "frozen" through millions of years of evolution [3,39]. After their existence was established both in vertebrates and invertebrates, researchers determined their role as cis-regulatory DNA; specifically, as enhancers to development regulatory genes mainly expressed in early embryonic development [14]. CNEs are not randomly distributed in the genome; they tend to form syntenic blocks containing an array of CNEs, a target gene and sometimes one or more bystander genes that are not affected by the regulatory input of the CNEs [34]. Research focusing on identifying distinct motifs similar to those found in protein coding sequences showed that CNEs are A+T-rich exhibiting a depletion in $\mathrm{A} / \mathrm{T}$ in their flanking regions [44]. Furthermore, Chiang et al. found that the most over-represented motif in CNEs is the core recognition sequence (TAAT) for homeodomain DNA-binding proteins, with TAATTA being the most prominent one [8]. 
Table 2: Binary Classification Experiments

\begin{tabular}{|l|l|l|}
\hline Experiment & Class 1 & Class 2 \\
\hline \#1 Human vs Worm & huUCNEs & WormUCNEs \\
\hline \#2 Human vs Insect & huUCNEs & Insect UCNEs \\
\hline \#3 Worm vs Insect & WormUCNEs & Insect UCNEs \\
\hline
\end{tabular}

4.1.2 Dataset Description \& Task Formulation. The dataset utilized, consists of conserved non-exonic sequences identified in both vertebrates (H. sapiens) and invertebrates (D. melanogaster, C. elegans) in FASTA format as described by Polychronopoulos et al. [35].

We perform three independent binary classification experiments displayed in Table 2. Since these sequences are highly similar, they provide an ideal starting point for our analysis.

\subsection{Preprocessing}

In order to apply NLP methods on DNA sequences, we have to convert them from the initial FASTA format to a text document resembling natural language. To do so, we perform the following steps:

(1) for each participating class:

- removal of FASTA headers

- k-mer extraction

- conversion to lowercase

(2) merging sequences from both classes into one file

The first step forms the "sentences" and "words" for each genomic class while the second combines all "sentences" into a single file.

For the tokenization of the DNA sequences into shorter subsequences analogous to natural language words we use a sliding window (step=1) of size $k=6$ to extract DNA k-mers. In the literature, these lengths vary greatly, as shown in Table 1, suggesting that the selection of $k$ highly depends on the use-case. In this paper, we have chosen 6-mers since Ultra-Conserved Non Coding Elements (UCNEs) are known to be enriched in "TAATTA" [8], of equal length. For this reason, this special attribute might be crucial for the identification of individual species. In contrast to other studies dealing with exonic sequences, k-mer sizes that are multiple of 3 can be selected for our analysis ${ }^{1}$.

\subsection{Embeddings}

We perform three distinct approaches for mapping the extracted 6-mers derived during the previous step to a vector space. These are the following:

(1) concatenation of nucleotide embeddings

(2) topic vectors

(3) centroid of word vectors

4.3.1 Concatenation of nucleotide embeddings. : This representation approach encompasses the biochemically inspired sparse embeddings introduced in the Related Work chapter 2.1. We perform zero-padding to force a consistent size to the "sentence" embeddings in order to subsequently apply ML techniques. These will function as the baseline for our subsequent comparisons as

${ }^{1}$ the latter are known to cause codon bias, a preference of specific 3-mers in some species these embeddings do not consider any form of context within the genomic sequences.

4.3.2 Topic vectors. With $\mathrm{LDA}$, it is possible to assign topic weights to natural language documents 2.4.1. We extend their usage for the extraction of topics from DNA sequences. Each sequence segment ${ }^{2}$ can be thought as a sentence and each 6-mer as a word. We map each DNA sentence to a vector

$$
V=<W_{s, T_{0}}, W_{s, T_{1}}, W_{s, T_{2}}, \ldots, W_{s, T_{m}}>
$$

where $s$ the set of k-mers, $W_{T_{i}} \in[0,1]$ the contribution of topic $i$ to $s$ and $m$ the number of topics.

We adopted the implementation of LDA with Gibbs sampling of MALLET [26], a popular Java framework for NLP used with JDK 1.8. For comparison purposes, we also produced topic vectors with lda2vec, resulting from the Document-Topic matrix after training for 1 epoch. For lda2vec, we used Python 3.6.9 and cupy-7.5.0 to speed up computations on a Tesla K80 GPU. Without loss of generality, and in order to match the dimensionality of GS, we chose $m=16$ topics. Thus, each sentence was mapped to a 16-dimensional embedding.

The resulting topic vectors are expected to introduce context into the sentence-level DNA embeddings by considering global patterns of k-mer occurrences. Furthermore, no padding is required and the input sequences are projected into a low-dimensional vector space.

4.3.3 Centroid of word vectors. lda2vec extracts word embeddings by combining word2vec and LDA. These embeddings are believed to be more context-sensitive than the original word2vec vectors, as they consider both local and global information [28]. First, we produce 30-dimensional and 100-dimensional embeddings with lda2vec (1 epoch, batch size $=32,16$ topics). Next, we represent each DNA sentence (subsequence containing k-mers) in the experiment by the centroid of all the k-mer word embeddings. The centroid can be thought as the representative word-vector for each sequence. Furthermore, with this approach we perform dimensionality reduction, and no padding is required, as all embeddings have the same size.

\subsection{Machine Learning Pipeline}

For each classification experiment and representation setup, we feed the sequence embeddings into multiple machine learning pipelines. The WEKA Java toolkit (version = 3.8.4) [13] was used to conduct the experiments, covering multiple popular learning algorithms including trees, neural networks, support-vector classifiers and regression-based approaches [6, 23, 33]. For each experiment, we perform 10 -fold cross-validation. The classes are perfectly balanced so over-sampling or under-sampling is not required. We choose Fmeasure as the evaluation metric, defined as $F_{1}=2 P R(P+R)$, where $P, R$ are the precision and recall of the classification experiment.

\section{RESULTS AND DISCUSSION}

The results of the classification experiments are summarized in Table 3. We observe that the concatenation of biochemically inspired nucleotide embeddings perform astonishingly well in the

\footnotetext{
${ }^{2}$ the set of k-mers corresponding to FASTA header
} 
Table 3: F-measure\% for all experiments and embeddings (higher is better). With bold, the two best-performing embeddings per classification. In parentheses, the best performing classifier for each embedding.

Abbreviations: Logistic=weka.classifiers.functions.Logistic, SMO:weka.classifiers.functions.SMO,Random Forest=weka.classifiers.trees.RandomForest [13]

\begin{tabular}{|c|c|c|c|c|c|}
\hline Approach & \multicolumn{2}{|c|}{ Embedding } & Human vs Worm & Human vs Insect & Worm vs Insect \\
\hline \multirow{2}{*}{ Concatenation of Nucleotide Embeddings } & \multicolumn{2}{|c|}{ Voss[43](Logistic) } & 67.3 & 66.15 & 92.2 \\
\hline & \multicolumn{2}{|c|}{ EIP[29] (Logistic) } & 62.65 & 61.35 & 93.2 \\
\hline \multirow[b]{2}{*}{ Topic Vectors } & \multicolumn{2}{|c|}{ LDA [5] (Logistic) } & 83.0 & 85.5 & 77.4 \\
\hline & lda2vec[28] & $\begin{array}{c}30 \mathrm{D} \\
(\mathrm{SMO})\end{array}$ & 53.7 & 51.1 & 53.0 \\
\hline \multirow[t]{2}{*}{ Centroid of Word Embeddings } & \multirow[t]{2}{*}{ lda2vec[28] } & $\begin{array}{c}\text { 30D } \\
\text { (Random Forest) }\end{array}$ & 71.1 & 62.1 & 71.2 \\
\hline & & $\begin{array}{c}100 \mathrm{D} \\
\text { (Logistic) }\end{array}$ & 70.6 & 71.6 & 71.8 \\
\hline Related Work & \multicolumn{2}{|c|}{ NGG $[12,35]$} & 77.7 & 82.1 & 70.9 \\
\hline
\end{tabular}

experiment involving Worm and Insect UCNEs but appears underwhelming in the rest. LDA, a context-based sequence embedding, with 16 topics fed into the logistic regression classifier, appears to perform consistently well in all experiments, and scores the highest F-measure for the experiment involving Human and Worm UCNEs.

A factor that is important to consider is that topic-representations do not encompass any kind of syntax related to the order of the sub-sequences in a sequence. The fact that they achieve competitive performance in the various experiments involving genomic sequences, is an indicator that the true order of nucleotides in a DNA sequence does not play a major role in its functionality. Furthermore, running the Topic Model multiple times varying the number of topics, can be a good way to capture both close-distance and long-distance interactions between the nucleotides. The intuition behind this is that if the number of topics is small, each topic will on average, span a larger area of the sequence than if the number of topics was very large.

On the other hand, in contrast to regular LDA with Gibbs sampling, the topic vectors resulting from lda2vec when trained with same number of topics achieve near-chance performance. This result suggests that LDA should be preferred over lda2vec when word-level information is ignored. Furthermore, increasing the embeddings dimensionality from 30 to 100 does not effect the final classification result. However, when considering the centroid of the word vectors, lda2vec manages to perform reasonably well and scores consistently close to $70 \% \mathrm{~F}$-measure score. We also observe that 100-dimensional embeddings perform considerably better than the 30-dimensional ones in the classification between Human and Insect UCNEs, but do not improve the performance in the other experiments. Compared to LDA, lda2vec centroids under-perform even though they utilize both shallow neural networks and topics' information.

Compared to NGGs which also take into account the neighborhoods of words, our representations and especially LDA manages to perform better in all conducted experiments. This difference might originate from information loss caused by the difference in feature vector dimensionality since the NGGs in the corresponding study [35] project the sequences into a 2D-space of similarities while LDA produces 16-dimensional embeddings. However, this performance gap between the two methods might be compensated by parameter-tuning in NGGs or by using 6-dimensional vectors to also include other similarity metrics, similarly to the study conducted by Kostagiolas et al.[22]. The difference between k-mer sizes might also be crucial for the final classification.

Furthermore, GS representations appear as very strong competitors for the task. GS consider context in terms of the dinucleotide odds ratio of subsequences and different species display different preferences. However, GS might not be suitable for intraspecies classification or when comparing sequences originating from closely-related organisms. Future work on the classification of mammalian or even primate UCNEs might put these representations at test.

lda2vec is an innovative approach which has recently been gaining a lot of popularity but did not manage to meet our expectations for the representation of DNA sequences. However, since lda2vec is an ensemble of approaches, it requires extensive parameter exploration and tuning. With careful adjustments, a representation like the word-vector centroids evaluated in this study might be able to compete with GS or LDA, although lda2vec is considerably slower and more complex than its competitors. Due to time and resource restrictions, the lda2vec representations were trained only on 1 epoch. Increasing the epochs might result in more efficient embeddings.

Overall, the representations that utilize high-level context perform well in all comparisons. The context-agnostic representations such as the nucleotide embeddings evaluated in this study, achieve impressive scores for one classification experiment ( Worm vs Insect) but lack behind in the rest of them. Thus, the results suggest that context-aware embeddings are more stable but more experimentation needs to take place to arrive at grounded results.

\section{CONCLUSIONS}

In this study we examined whether embeddings that utilize context can improve the classification of DNA sequences. We performed multiple classification experiments involving conserved non-coding 
elements from diverse species, which exhibit a high degree of similarity. We evaluated three different families for embeddings, which are either formed at the nucleotide-level (sparse), at the k-mer level (lda2vec) or at the sequence-level (LDA). To apply NLP techniques, we treated DNA sequences as natural language documents by defining letters, words, sentences or topics.

Our experiments, along with those conducted before, like $\mathrm{N}$ Gram Graphs and Genomic Signatures, provide evidence that ignoring the exact order of nucleotides is not detrimental as bag-of-word approaches perform significantly well. Simple embeddings like Voss and Paired-Numeric performed surprisingly well in the experiment involving Worm and Insect UCNEs.

To our knowledge, LDA and lda2vec along with ML were applied for the first time on UCNEs. The results suggest that both methods can provide useful embeddings for DNA sequence classification. However, lda2vec embeddings appear useful only when considering word-level information as the topic vectors produced by lda2vec achieve baseline performance.

The results provided in this study appear promising and suggest that high-level context is a crucial factor for the representation of conserved elements. We strongly believe that further parameter tuning and experimentation with the presented embeddings will be able to uncover patterns that affect the quality of DNA embeddings. Lastly, the topics produced by LDA and the embeddings from lda2vec can provide insight into the structure of the conserved elements and aid biological discovery.

\section{REFERENCES}

[1] Vishal Agarwal, N Reddy, and Ashish Anand. 2019. Unsupervised Representation Learning of DNA Sequences. arXiv preprint arXiv:1906.03087 (2019).

[2] Mahmood Akhtar, Julien Epps, and Eliathamby Ambikairajah. 2007. On DNA numerical representations for period-3 based exon prediction. In 2007 IEEE international workshop on genomic signal processing and statistics. IEEE, 1-4.

[3] Gill Bejerano, Michael Pheasant, Igor Makunin, Stuart Stephen, W James Kent, John S Mattick, and David Haussler. 2004. Ultraconserved elements in the human genome. Science 304, 5675 (2004), 1321-1325.

[4] Richard Bellman. 1958. Dynamic programming and stochastic control processes. Information and Control 1, 3 (1958), 228 - 239. https://doi.org/10.1016/S00199958(58)80003-0

[5] David M. Blei. 2012. Probabilistic topic models. Commun. ACM 55, 4 (April 2012), 77. https://doi.org/10.1145/2133806.2133826

[6] Leo Breiman. 2001. Random forests. Machine learning 45, 1 (2001), 5-32. Publisher: Springer.

[7] Betty Yee Man Cheng, Jaime G Carbonell, and Judith Klein-Seetharaman. 2005. Protein classification based on text document classification techniques. Proteins: Structure, Function, and Bioinformatics 58, 4 (2005), 955-970.

[8] Charleston WK Chiang, Adnan Derti, Daniel Schwartz, Michael F Chou, Joel N Hirschhorn, and others. 2008. Ultraconserved elements: analyses of dosage sensitivity, motifs and boundaries. Genetics 180, 4 (2008), 2277-2293.

[9] Madhavi Ganapathiraju, Deborah Weisser, Roni Rosenfeld, Jaime Carbonell, Raj Reddy, and Judith Klein-Seetharaman. 2002. Comparative n-gram analysis of whole-genome protein sequences. In Proceedings of the second international conference on Human Language Technology Research. Morgan Kaufmann Publishers Inc., 76-81.

[10] Prakhar Ganesh, Gaurav Gupta, Shubhi Saini, and Kolin Paul. 2018. Nucl2Vec: Local alignment of DNA sequences using Distributed Vector Representation. BioRxiv (2018), 401851. Publisher: Cold Spring Harbor Laboratory.

[11] Stuart Geman and Donald Geman. 1984. Stochastic relaxation, Gibbs distributions, and the Bayesian restoration of images. IEEE Transactions on pattern analysis and machine intelligence 6 (1984), 721-741. Publisher: IEEE.

[12] George Giannakopoulos, Vangelis Karkaletsis, George Vouros, and Panagiotis Stamatopoulos. 2008. Summarization System Evaluation Revisited: N-gram Graphs. ACM Trans. Speech Lang. Process. 5, 3 (Oct. 2008), 5:1-5:39. https: //doi.org/10.1145/1410358.1410359

[13] Mark Hall, Eibe Frank, Geoffrey Holmes, Bernhard Pfahringer, Peter Reutemann, and Ian $\mathrm{H}$ Witten. 2009. The WEKA data mining software: an update. ACM SIGKDD explorations newsletter 11, 1 (2009), 10-18. Publisher: ACM New York,
NY, USA.

[14] Nathan Harmston, Elizabeth Ing-Simmons, Ge Tan, Malcolm Perry, Matthias Merkenschlager, and Boris Lenhard. 2017. Topologically associating domains are ancient features that coincide with Metazoan clusters of extreme noncoding conservation. Nature communications 8, 1 (2017), 1-13.

[15] Zellig S. Harris. 1954. Distributional Structure. WORD 10, 2-3 (1954), 146-162. https://doi.org/10.1080/00437956.1954.11659520

[16] Todd Holden, R Subramaniam, R Sullivan, E Cheung, C Schneider, G Tremberger Jr, A Flamholz, DH Lieberman, and TD Cheung. 2007. ATCG nucleotide fluctuation of Deinococcus radiodurans radiation genes. In Instruments, Methods, and Missions for Astrobiology X, Vol. 6694. International Society for Optics and Photonics, 669417

[17] Samuel Kariin and Chris Burge. 1995. Dinucleotide relative abundance extremes: a genomic signature. Trends in Genetics 11, 7 (1995), 283 - 290. https://doi.org/ 10.1016/S0168-9525(00)89076-9

[18] Samuel Karlin and Lon R Cardon. 1994. Computational DNA sequence analysis. Annual review of microbiology 48, 1 (1994), 619-654.

[19] S Karlin, J Mrázek, and A M Campbell. 1997. Compositional biases of bacterial genomes and evolutionary implications. Fournal of Bacteriology 179, 12 (June 1997), 3899-3913. https://doi.org/10.1128/jb.179.12.3899-3913.1997

[20] Jong Yong Kim and John Shawe-Taylor. 1994. Fast string matching using an n-gram algorithm. Software: Practice and Experience 24, 1 (1994), 79-88.

[21] Min-Soo Kim, Kyu-Young Whang, Jae-Gil Lee, and Min-Jae Lee. 2005. n-gram/21: A space and time efficient two-level n-gram inverted index structure. In Proceedings of the 31st international conference on Very large data bases. VLDB Endowment, 325-336.

[22] Nikos Kostagiolas, Nikiforos Pittaras, Christoforos Nikolaou, and George Giannakopoulos. 2018. Exploring different sequence representations and classification methods for the prediction of nucleosome positioning. bioRxiv (2018), 482612.

[23] Saskia Le Cessie and Johannes C Van Houwelingen. 1992. Ridge estimators in logistic regression. Journal of the Royal Statistical Society: Series C (Applied Statistics) 41, 1 (1992), 191-201. Publisher: Wiley Online Library.

[24] Omer Levy and Yoav Goldberg. 2014. Neural word embedding as implicit matrix factorization. In Advances in neural information processing systems. 2177-2185.

[25] RN Mantegna, SV Buldyrev, AL Goldberger, S Havlin, C-K Peng, M Simons, and HE Stanley. 1995. Systematic analysis of coding and noncoding DNA sequences using methods of statistical linguistics. Physical Review E 52, 3 (1995), 2939.

[26] Andrew Kachites McCallum. 2002. MALLET: A Machine Learning for Language Toolkit. (2002).

[27] Tomas Mikolov, Kai Chen, Greg Corrado, and Jeffrey Dean. 2013. Efficient Estimation of Word Representations in Vector Space. arXiv:1301.3781 [cs] (Jan. 2013). http://arxiv.org/abs/1301.3781

[28] Christopher E Moody. 2016. Mixing dirichlet topic models and word embeddings to make lda2vec. arXiv preprint arXiv:1605.02019 (2016).

[29] Achuthsankar S Nair and Sivarama Pillai Sreenadhan. 2006. A coding measure scheme employing electron-ion interaction pseudopotential (EIIP). Bioinformation 1, 6 (2006), 197. Publisher: Biomedical Informatics Publishing Group.

[30] Saul B Needleman and Christian D Wunsch. 1970. A general method applicable to the search for similarities in the amino acid sequence of two proteins. Fournal of molecular biology 48, 3 (1970), 443-453. Publisher: Academic Press.

[31] Patrick Ng. 2017. dna2vec: Consistent vector representations of variable-length k-mers. arXiv preprint arXiv:1701.06279 (2017).

[32] Marcelo A Nobrega, Ivan Ovcharenko, Veena Afzal, and Edward M Rubin. 2003. Scanning human gene deserts for long-range enhancers. Science 302, 5644 (2003), 413-413. Publisher: American Association for the Advancement of Science.

[33] John C Platt. 1999. Using analytic QP and sparseness to speed training of support vector machines. In Advances in neural information processing systems. 557-563.

[34] Dimitris Polychronopoulos, James W. D. King, Alexander J. Nash, Ge Tan, and Boris Lenhard. 2017. Conserved non-coding elements: developmental gene regulation meets genome organization. Nucleic Acids Research 45, 22 (Dec. 2017), 12611-12624. https://doi.org/10.1093/nar/gkx1074

[35] Dimitris Polychronopoulos, Anastasia Krithara, Christoforos Nikolaou, Giorgos Paliouras, Yannis Almirantis, and George Giannakopoulos. 2014. Analysis and Classification of Constrained DNA Elements with N-gram Graphs and Genomic Signatures. In Algorithms for Computational Biology, David Hutchison, Takeo Kanade, Josef Kittler, Jon M. Kleinberg, Alfred Kobsa, Friedemann Mattern, John C. Mitchell, Moni Naor, Oscar Nierstrasz, C. Pandu Rangan, Bernhard Steffen, Demetri Terzopoulos, Doug Tygar, Gerhard Weikum, Adrian-Horia Dediu, Carlos Martín-Vide, and Bianca Truthe (Eds.). Vol. 8542. Springer International Publishing, Cham, 220-234. https://doi.org/10.1007/978-3-319-07953-0_18

[36] David T Pride, Richard J Meinersmann, Trudy M Wassenaar, and Martin J Blaser. 2003. Evolutionary implications of microbial genome tetranucleotide frequency biases. Genome research 13, 2 (2003), 145-158

[37] Ji Qi, Hong Luo, and Bailin Hao. 2004. CVTree: a phylogenetic tree reconstruction tool based on whole genomes. Nucleic acids research 32, suppl_2 (2004), W45W47.

[38] Nitin Sabherwal, Fiona Bangs, Ralph Röth, Birgit Weiss, Karin Jantz, Eva Tiecke, Georg K Hinkel, Christiane Spaich, Berthold P Hauffa, Hetty van der Kamp, 
and others. 2007. Long-range conserved non-coding SHOX sequences regulate expression in developing chicken limb and are associated with short stature phenotypes in human patients. Human molecular genetics 16, 2 (2007), 210-222. Publisher: Oxford University Press.

[39] Albin Sandelin, Peter Bailey, Sara Bruce, Pär G Engström, Joanna M Klos, Wyeth W Wasserman, Johan Ericson, and Boris Lenhard. 2004. Arrays of ultraconserved non-coding regions span the loci of key developmental genes in vertebrate genomes. BMC genomics 5, 1 (2004), 99.

[40] Victor V Solovyev and Kira S Makarova. 1993. A novel method of protein sequence classification based on oligopeptide frequency analysis and its application to search for functional sites and to domain localization. Bioinformatics 9, 1 (1993),
$17-24$.

[41] Mark Steyvers and Tom Griffiths. 2017. Probabilistic Topic Models. (2017), 15.

[42] Richard C Tillquist and Manuel E Lladser. 2019. Low-dimensional representation of genomic sequences. Fournal of mathematical biology 79, 1 (2019), 1-29. Publisher: Springer.

[43] Richard F Voss. 1992. Evolution of long-range fractal correlations and 1/f noise in DNA base sequences. Physical review letters 68, 25 (1992), 3805. Publisher: APS.

[44] Klaudia Walter, Irina Abnizova, Greg Elgar, and Walter R Gilks. 2005. Striking nucleotide frequency pattern at the borders of highly conserved vertebrate noncoding sequences. Trends in Genetics 21, 8 (2005), 436-440. 